\title{
Erratum to: Autonomic Communication
}

\author{
Ioannis Stavrakakis ${ }^{1}$ and Michael Smirnov ${ }^{2}$ \\ 1 National and Kapodistrian University of Athens, Department of Informatics \\ and Telecommunications, Panepistimiopolis, Ilisia, 15784 Athens, Greece \\ ioannis@di.uoa.gr \\ 2 Fraunhofer Institut FOKUS, Kaiserin-Augusta Allee 31, 10589 Berlin, Germany \\ smirnov@fokus.fraunhofer.de
}

\section{Erratum to:}

\section{Stavrakakis and M. Smirnov (Eds.) Autonomic Communication DOI: $10.1007 / 978-3-540-32993-0$}

The book was inadvertently published with an incorrect name of the copyright holder. The name of the copyright holder for this book is: (C) Springer-Verlag Berlin Heidelberg. The book has been updated with the changes.

The updated original online version for this book can be found at DOI: $10.1007 / 978-3-540-32993-0$ 